\title{
ShareDay: A Novel Lifelog Management System for Group Sharing
}

\author{
Lijuan Marissa Zhou ${ }^{1}$, Niamh Caprani ${ }^{1,2}$, Cathal Gurrin ${ }^{1}$, \\ and Noel E. O'Connor ${ }^{2}$ \\ 1 CLARITY: Centre for Sensor Web Technologies, Dublin City University \\ 2 School of Electronic Engineering, Dublin City University \\ \{mzhou, ncaprani, cgurrin\}@computing.dcu.ie, oconnorn@eeng.dcu.ie
}

\begin{abstract}
Lifelogging is the automatic capture of daily activities using environmental and wearable sensors such as MobilePhone/SenseCam. The potential to capture such a large data collection presents many challenges, including data analysis, visualisation and motivating users of different ages and technology experience to lifelog. In this paper, we present a new generation of lifelog system to support reminiscence through incorporating event segmentation and group sharing.
\end{abstract}

Keywords: Multimedia System, Group Sharing, Lifelogging, Touch Screen.

\section{Introduction}

With the recent availability of wearable sensing technologies and an acceptance of personal data gathering and on-line social sharing (e.g, on Facebook timeline), lifelogging has become a mainstream research topic. We now have the ability to gather and store large volumes of personal data using an inexpensive smart phone. However, with many available lifelogging tools, how to collect, organize and represent lifelog data is still under much discussion [12].

Furthermore, people have always collected mementos over lifetime. With the digitization of mementos (photos and videos etc.), researchers have begun to realize the benefit of this to support reminiscence [3]. Sharing digital information is already commonplace, through emails, mobile phones and social networks. However, sharing lifelog data between family members, to our knowledge, has not yet been looked at. Shared reminiscence between family members can serve many functions such as maintaining memories of past relatives, creating bonds and teaching younger family members from the elders' experiences. We believe that sharing lifelogs within a family would enrich reminiscence and story-telling. In this paper we describe a novel software system to support sharing lifelog.

\section{ShareDay}

A previous study on intergenerational sharing [4] has shown that both older and younger people were more likely to wear a lifelogging device for the purpose of

S. Li et al. (Eds.): MMM 2013, Part II, LNCS 7733, pp. 490-492, 2013.

(C) Springer-Verlag Berlin Heidelberg 2013 
sharing images rather than simply wearing a lifelog device for private browsing or reminiscence. ShareDay was designed to support browsing and sharing through lifelogs on cross-platforms. To support family reminiscence we have designed the system to be used on a touch screen device displayed in a communal area at home so that all family members can upload, view and share their lifelogs. Users can also view specific person's shared data by clicking on their profile (see Figure 1). The lifelog data can be viewed in two modes: personal and family. In family view, all group members can see all daily events and images in an overall, shared or favourite view. In personal view, only the logged-user can upload data and view their data. If logged, users can manage (share, mark as favourite or delete) and browse their visual lifelogs. There are three main functions incorporated into the ShareDay system: managing personal data, shared data and favourite data. Fig. 1 is the main view of the system, which shows all events gathered by all family members in that day. These functions will be described as follows.

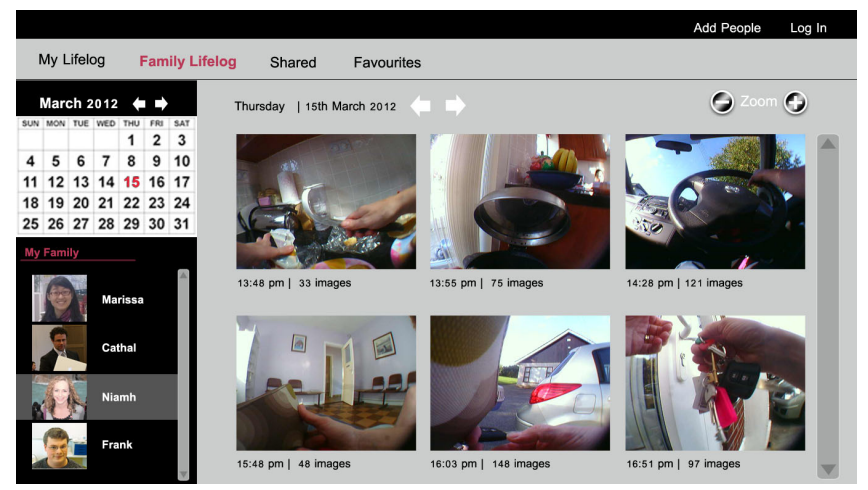

Fig. 1. ShareDay System Overview: Family View

\subsection{Managing Personal Lifelog Data}

To collect a personal lifelog, the user must initially capture images and upload them to the system. Visual lifelogs present a challenge for developers as they need to represent the users day accurately and in a user-friendly manner, without requiring the user to browse through up to 5,000 images per day. We integrated an event segmentation model [5], which organizes a sequence of SenseCam images into a set of events. Events represent daily activities such as walking, eating, shopping, talking, etc. Keyframe images representing events are selected and displayed for each event, with six large keyframe images being selected for each event. When a user is logged in they can manage (share, favourite or delete) and browse through their visual lifelog. With regard to sharing, logged user can share/mark favourite their daily events/images, so other family members can see shared data, which is designed under the concern of privacy. 


\subsection{Sharing Lifelog Data}

The initial screen of the system displays shared lifelog of each family member. The user can touch on the name of their family member to view lifelogs. A user can also browse through shared lifelogs organized chronologically by touching the Shared tab. Group sharing to support family reminiscence is the primary aim of the proposed lifelog management system. However, we also wanted to ensure that users had control over their own lifelogs as the content can be extremely personal. To accommodate for this users can select to share images/events when they are logged into their accounts. These images will automatically be transferred to communal lifelog data set which all members of the family can view.

\subsection{Favourite Lifelog Data}

In our previous studies the participants reported that when they wanted to share images they had difficulty finding the images due to the vast data set accumulated 24]. An easy fix for this was to provide users with a Favourites button. These selected images are automatically duplicated into a favourites folder which users can find on the main menu bar.

\section{Discussion and Conclusion}

We demonstrate a novel lifelogging system for group sharing, which emphasizes on sociability of SenseCam users. In future, we will conduct more user studies on influence of group sharing/favourite to personal lifelog and reminiscence.

Acknowledgement. This research is supported by SFI(11/RFP.1/CMS/3283) and Embark Initiative.

\section{References}

1. Caprani, N., Doherty, A.R., Lee, H., Smeaton, A.F., O'Connor, N.E., Gurrin, C.: Designing a touch-screen sensecam browser to support an aging population. In: CHI Extended Abstracts, pp. 4291-4296 (2010)

2. Zhou, L.M., Gurrin, C.: A survey on life logging data capture. In: SenseCam 2012: 3rd Annual Symposium, SenseCam 2012 (2012)

3. Peesapati, S.T., Schwanda, V., Schultz, J., Lepage, M., Yae Jeong, S., Cosley, D.: Pensieve: supporting everyday reminiscence. In: CHI, pp. 2027-2036 (2010)

4. Caprani, N., Gurrin, C., O'Connor, N.E.: Sharing as a motivation for lifelogging. In: SenseCam 2012: 3rd Annual Symposium, SenseCam 2012 (2012)

5. Doherty, A.R., Conaire, C.O., Blighe, M., Smeaton, A.F., O'Connor, N.E.: Combining image descriptors to effectively retrieve events from visual lifelogs. In: Multimedia Information Retrieval, pp. 10-17 (2008) 